\title{
Passive leg raising for predicting fluid responsiveness: a systematic review and meta-analysis
}

\author{
X Monnet ${ }^{*}$, J-L Teboul \\ From ESICM LIVES 2015 \\ Berlin, Germany. 3-7 October 2015
}

\section{Introduction}

The passive leg raising (PLR) test has been proposed to predict fluid responsiveness in patients with acute circulatory failure. We reviewed and meta-analysed the studies that investigated the PLR-induced changes in cardiac output (CO) or surrogates and the PLR-induced changes in arterial pulse pressure (PP) as predictors of fluid responsiveness.

\section{Methods}

MEDLINE, EMBASE and Cochrane Database of Systematic Reviews were screened for relevant original and review article. Studies quality was assessed with the QUADAS-2 scale.

\section{Results}

Twenty-two studies including a total of 975 patients responded to the selection criteria for the PLR-induced changes in $\mathrm{CO}$. In these studies, $\mathrm{CO}$ was measured by echocardiography in 7 studies, transpulmonary thermodilution in 7 studies, bioreactance in 4 studies, oesophageal Doppler in 3 studies, pulmonary artery catheter in one study and suprasternal Doppler in one study. Among those 22 studies, 9 including a total of 432 patients responded to the selection criteria for the PLRinduced changes in PP. Data are reported as mean (95\% confidence interval) or mean \pm standard deviation. All studies but two were conducted in adults. The pooled correlation between the PLR-induced changes in $\mathrm{CO}$ and the volume expansion-induced changes in $\mathrm{CO}$ was 0.73 (0.69-0.77). For the PLR-induced changes in CO, the pooled sensitivity was $0.81(0.84-0.87)$ and the pooled specificity was $0.90(0.87-0.92)$. The pooled area under the Receiver Operating Characteristics (ROC) curve was $0.95 \pm 0.01$. The mean of the best threshold was a PLR-induced increase in CO of more than $10 \pm$ $2 \%$. For the PLR-induced changes in PP, the pooled sensitivity was $0.51(0.44-0.58)$ and the pooled specificity was $0.84(0.78-0.89)$. The pooled area under the ROC curve was $0.79 \pm 0.04$ ( $\mathrm{p}<0.01$ vs. the area under the ROC curve for the PLR-induced changes in $\mathrm{CO}$ ). The mean of the best threshold was a PLR-induced increase in PP of more than $12 \pm 4 \%$.

\section{Conclusions}

The PLR test is highly reliable in predicting the response of $\mathrm{CO}$ to volume expansion in patients with acute circulatory failure. Its predictive value is significantly better when its effects are assessed by measuring $\mathrm{CO}$ or a surrogate of $\mathrm{CO}$ than when measuring $\mathrm{PP}$, in particular with a lower specificity.

Published: 1 October 2015

doi:10.1186/2197-425X-3-S1-A587

Cite this article as: Monnet and Teboul: Passive leg raising for

predicting fluid responsiveness: a systematic review and meta-analysis.

Intensive Care Medicine Experimental 2015 3(Suppl 1):A587. 